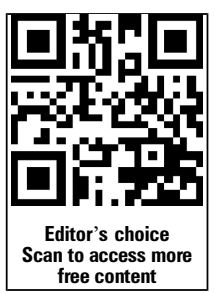

Knight Cardiovascular Institute, Oregon Health Science University, Portland, Oregon, USA

${ }^{2}$ Division of Cardiology, Thorax Center, Groningen, The Netherlands

${ }^{3}$ Division of Cardiology, Erasmus University Medical Centre, University Medical Centre, Rotterdam, The Netherlands

${ }^{4}$ Division of Cardiology, University of British Columbia, Providence Health Care,

St. Paul's Hospital, Vancouver, Canada

Division of Cardiology, University of Toronto, Mount Sinai Hospital and University Health Network, Toronto, Canada

${ }^{6}$ Department of Obstetrics and Gynaecology, University Medical Center Utrecht, University of Utrecht, Utrecht, The Netherlands ${ }^{7}$ Division of Cardiology, University of Western Ontario, University Hospital, London, Canada

Correspondence to Dr Candice K Silversides, Mount Sinai Hospital, OPG Building, 700 University Avenue, 3rd Floor, Room 9-132, Toronto, Ontario Canada M5G 2N2; candice.silversides@uhn.ca

Received 29 August 2014 Revised 7 November 2014 Accepted 5 December 2014 Published Online First 6 January 2015

\section{Linked}

- http://dx.doi.org/10.1136/ heartjnl-2014-307289

\section{CrossMark}

\section{To cite: Burchill LJ,}

Lameijer H, Roos-

Hesselink JW, et al. Heart 2015;101:525-529.

\title{
Pregnancy risks in women with pre-existing coronary artery disease, or following acute coronary syndrome
}

\author{
Luke J Burchill, ${ }^{1}$ Heleen Lameijer, ${ }^{2}$ Jolien W Roos-Hesselink, ${ }^{3}$ Jasmine Grewal, ${ }^{4}$ \\ Titia PE Ruys, ${ }^{3}$ Julia D Kulikowski, ${ }^{5}$ Laura A Burchill, ${ }^{5}$ M A Oudijk, ${ }^{6}$ Rachel M Wald, ${ }^{5}$ \\ Jack M Colman, ${ }^{5}$ Samuel C Siu, ${ }^{6}$ Petronella G Pieper, ${ }^{2}$ Candice K Silversides ${ }^{5}$
}

\begin{abstract}
Objective The objective of this study was to determine outcomes in pregnant women with pre-existing coronary artery disease (CAD) or following an acute coronary syndrome (ACS) including myocardial infarction (MI).

Background The physiological changes of pregnancy can contribute to myocardial ischaemia. The pregnancy risk for women with pre-established CAD or a history of ACS/MI is not well studied.

Methods This was a retrospective multicentre study. Adverse maternal cardiac, obstetric and fetal/neonatal events were examined. The primary outcome was a composite endpoint of cardiac arrest, ACS/MI, ventricular arrhythmia or congestive heart failure. The prevalence of new or progressive angina during pregnancy was also examined.
\end{abstract}

Results Fifty pregnancies in 43 women (mean age 35 \pm 5 years) were included. Coronary atherosclerosis (40\%) and coronary thrombus (36\%) were the most common underlying diagnoses. The primary outcome occurred in $10 \%(5 / 50)$ of pregnancies and included one maternal death secondary to cardiac arrest. Other events included ACS/MI (3/50) and heart failure (1/50). New or progressive angina occurred in 18\% of pregnancies. Ischaemic complications of any type (new or progressive angina, ACS/MI, ventricular arrhythmia, cardiac arrest) occurred more commonly in women with coronary atherosclerosis compared with those without (50\% vs $10 \%, p=0.003)$. A high rate of adverse obstetric (16\%) and fetal/neonatal (30\%) events was observed.

Conclusions Pregnant women with pre-existing $C A D$ or ACS/MI before pregnancy are at increased risk of adverse events during pregnancy. Those with coronary atherosclerosis are at highest risk of adverse maternal cardiac events due to myocardial ischaemia during pregnancy.

Current trends have led to an older age at the time of childbirth and increases in the prevalence of maternal diabetes, obesity and hypertension. These factors have contributed to a rising number of women of childbearing age with pre-existing coronary disease. ${ }^{1-3}$ Ischaemic cardiac events, such as acute myocardial infarction (MI) have been associated with high maternal and fetal mortality. ${ }^{4-6}$ While early case reports on pregnancy risk in women with pre-existing coronary artery disease (CAD) reported high maternal mortality, ${ }^{7}$ subsequent case series reported improved maternal outcomes. ${ }^{8}{ }^{9}$ While many women with pre-existing CAD or a history of acute coronary syndrome (ACS) will consider pregnancy, the pregnancy risk for these women is not defined. The objective of this study was to determine maternal and fetal/neonatal outcomes in a large contemporary group of pregnant women with a history of CAD or ACS/MI prior to pregnancy.

\section{METHODS}

\section{Study population}

This was a multicentre review of pregnancies in women seen between 1995 and 2012 at one of the participating centres (University of Toronto, Toronto, Canada; University of British Columbia, Vancouver, Canada; Erasmus University, Rotterdam, The Netherlands; University Medical Centre, Groningen, The Netherlands; Academic Medical Center, Amsterdam, The Netherlands; and University Medical Center Utrecht, Utrecht, the Netherlands). This study was approved by the institutional review boards of the participating centres.

Cases were identified by systematic review of each institution's clinical database at each of the study centres. Inclusion criteria encompassed women referred with a history of CAD or an ACS, including $\mathrm{MI}$, preceding pregnancy. ACS was defined as chest pain, electrocardiographic changes and abnormal biomarkers not meeting criteria for MI. MI was defined as elevated cardiac biomarkers (CK-MB, troponin) with at least one value above the 99th percentile and one of the following: symptoms of ischaemia, electrocardiographic changes, imaging evidence of new regional wall-motion abnormality or loss of viable myocardium, and/or identification of intracoronary thrombus by angiography. ${ }^{10}$ Unstable angina and coronary spasm were clinical diagnoses made by the treating physician at the time of the previous clinical encounter. Women with first presentation of an ACS (including coronary dissection) during a pregnancy were not included. Women with Kawasaki disease or anomalous coronary arteries without history of ischaemic events were not included.

\section{Baseline data}

Demographic and clinical data were obtained from chart review and included maternal and gestational age, parity, earlier interventions, comorbidities (ie, 
smoking history, diabetes, hypertension, inherited thrombophilia), New York Heart Association (NYHA) functional class, and medications. Assessment of LV systolic function was based on clinical reports and classified as: normal function (LVEF $\geq 55 \%)$, mild $(45 \%-54 \%)$, moderate $(30 \%-44 \%)$ or severe $(<30 \%)$ systolic dysfunction. ${ }^{11}$

\section{Outcomes}

The primary maternal cardiac outcomes of interest were cardiac arrest or cardiac death, sustained or symptomatic ventricular tachycardia, ACS, MI and pulmonary oedema. In one woman, a planned elective coronary artery bypass operation was delayed because of an unexpected pregnancy. The elective bypass took place postpartum, but was not considered an endpoint. Other outcomes of interest included new/progressive angina, thromboembolism and atrial arrhythmia requiring treatment. Cardiac adverse events were further classified as ischaemic (unstable angina, MI, ventricular tachyarrhythmia or cardiac arrest) or non-ischaemic.

Adverse fetal and/or neonatal events included premature birth ( $<37$ weeks gestation), low birth weight $(<2500 \mathrm{~g})$, intrauterine growth retardation (fetal weight $<10$ th percentile for gestational age), respiratory distress syndrome, intraventricular haemorrhage, spontaneous abortion ( $<24$ weeks) and fetal or neonatal death.

Adverse obstetric events included pre-eclampsia (diagnosed by the treating physician) and postpartum haemorrhage (blood loss $>500 \mathrm{~mL}$ after vaginal delivery or $>1000 \mathrm{~mL}$ after caesarean section). ${ }^{12}$

\section{Statistics}

All data were analysed using SPSS V.16.0 (SPSS, Chicago, Illinois, USA). Normally distributed continuous variables were represented as a mean $\pm \mathrm{SD}$, and categorical variables were represented as a frequency. The differences between groups were determined using Student $t$ test or $\chi^{2}$ test; $\mathrm{p}<0.05$ (twosided) was considered to be significant.

\section{RESULTS}

During the study period, 50 pregnancies were identified in 43 women with pre-existing CAD or a history of ACS/MI prior to pregnancy. Baseline characteristics are summarised in table 1.

The majority of women (74\%) were multiparous, and the mean study age was $34 \pm 5$ years. Established risk factors for coronary disease were identified in $80 \%$ of women, the most common being a history of cigarette smoking, present in almost $60 \%$.

The most common diagnoses were atherosclerosis $(n=20)$ and intracoronary thrombus $(\mathrm{n}=18)$. Disorders of hypercoaguability were documented in seven women: Factor $\mathrm{V}$ Leiden mutation $(n=3)$, antiphospholipid syndrome $(n=3)$ and protein $S$ deficiency $(n=1)$. Less common findings included congenital fibroelastosis of the left main $(n=1)$ and coronary spasm $(n=5)$. Cardiac catheterisation had been performed prior to pregnancy in the majority of cases $(n=39,78 \%)$. Coronary atherosclerosis was diagnosed in 12 women at the time of cardiac catheterisation; these women were older ( $36 \pm 5$ years), more likely to be multiparous $(p=0.01)$ and to have multivessel disease $(p<0.0001)$ versus women without atherosclerosis. Women diagnosed with intracoronary thrombus were younger and were more likely to be anticoagulated $(\mathrm{p}<0.0001)$. Eleven women did not undergo cardiac catheterisation after presenting with ACS. Coronary spasm was diagnosed by the treating physician as the aetiology of ACS in five women on the basis of their clinical presentation and investigation results. Aetiology was not
Table 1 Baseline characteristics

\begin{tabular}{|c|c|}
\hline \multicolumn{2}{|l|}{ Demographic factors } \\
\hline Women & 43 \\
\hline Pregnancies & 50 \\
\hline Mean age at delivery (years) & $35 \pm 5$ \\
\hline Age range (years) & $27-47$ \\
\hline Multiparous & $37(74)$ \\
\hline \multicolumn{2}{|l|}{ Cardiac diagnosis } \\
\hline Atherosclerosis & 20 \\
\hline Coronary thrombus or embolism & 18 \\
\hline Coronary spasm & 5 \\
\hline Acute coronary syndrome* & 6 \\
\hline \multicolumn{2}{|l|}{ Pre-existing coronary risk factors } \\
\hline$\geq 1$ risk factor & $41(82)$ \\
\hline Smoking & $11(22)$ \\
\hline Family history & $22(44)$ \\
\hline Hypertension & $18(36)$ \\
\hline Dyslipidaemia & $10(20)$ \\
\hline Obesity & $5(10)$ \\
\hline Diabetes & $4(8)$ \\
\hline Thrombophiliat & $7(14)$ \\
\hline \multicolumn{2}{|c|}{ Cardiac medications at first antenatal visit $n=44$} \\
\hline Any medication & 47 (94) \\
\hline Aspirin/clopidogrel & 28 \\
\hline$\beta$-blocker & 19 \\
\hline Heparin & 8 \\
\hline Warfarin & 6 \\
\hline ACE inhibitor & 8 \\
\hline \multicolumn{2}{|c|}{ NYHA functional class at first antenatal visit } \\
\hline 1 & 42 \\
\hline II-IV & 8 \\
\hline \multicolumn{2}{|l|}{ LV systolic function§ $n=45$} \\
\hline Normal & $27(54)$ \\
\hline Mildly reduced & $13(26)$ \\
\hline Moderately reduced & $5(10)$ \\
\hline \multicolumn{2}{|c|}{$\begin{array}{l}\text { Note: Coronary anatomy findings are mutually exclusive. } \\
\text { *Acute coronary syndrome documented but aetiology not specified. } \\
\text { †Thrombophilia (Factor } V \text { Leiden }(n=3) \text {, antiphospholipid syndrome }(n=3) \text {, protein } S \\
\text { deficiency }(n=1)) \text { confirmed with genetic/lab testing. } \\
\text { ‡Medications not documented in six pregnancies. } \\
\text { §LV function assessed by transthoracic echocardiogram at first antenatal visit. } \\
\text { NYHA, New York Heart Association. }\end{array}$} \\
\hline
\end{tabular}

documented in six women who met criteria for a history of ACS prior to pregnancy, but did not undergo cardiac catheterisation.

Previous cardiac interventions were documented in 54\% (27/ $50)$ and included balloon angioplasty $(n=5)$, coronary stent implantation $(n=13)$, thrombolysis $(n=6)$ and coronary artery bypass graft $(\mathrm{CABG})$ surgery $(n=3)$. Aspirin was the most common cardiac medication $(n=27,54 \%)$, followed by anticoagulants $(n=12,24 \%), \beta$-blockers $(n=38,19 \%)$ and statins $(n=4,8 \%)$; the latter was stopped during pregnancy. ACE inhibitors had been used by $20 \%(10 / 50)$; these were discontinued prior to pregnancy in six women, and in the first trimester at the time of their first obstetric visit/cardiac review in the remaining four women.

\section{Maternal cardiac outcomes}

The primary outcome was observed in 10\% $(n=5)$ of pregnancies (tables 2 and 3, figure 1). There was one sudden death, 8 weeks postpartum, presumed secondary to ventricular 
Table 2 Adverse maternal cardiac events during pregnancy

\begin{tabular}{|c|c|c|c|c|c|c|c|c|}
\hline & \multicolumn{4}{|c|}{ Primary cardiovascular endpoint } & \multicolumn{4}{|c|}{ Secondary cardiovascular endpoint } \\
\hline & Any primary event & $\begin{array}{l}\text { Cardiac arrest/ventricular } \\
\text { arrhythmia }\end{array}$ & ACS/MI & Heart failure & Any secondary event & Angina & Stroke/PE & Atrial arrhythmia* \\
\hline Total & $5(10)$ & 1 & 3 & 1 & $12(24)$ & 9 & 2 & 1 \\
\hline
\end{tabular}

arrhythmia in a woman with a history of MI and moderate LV systolic dysfunction. Two women with atherosclerotic coronary disease sustained MI during the postpartum period (at 4 and 8 weeks postpartum). Cardiac catheterisation revealed significant CAD leading to percutaneous coronary intervention in both patients. A 42-year-old woman with a history of coronary bypass graft surgery and normal LVEF presented with acute pulmonary oedema at 33 weeks gestation. Pulmonary oedema was attributed to a combination of factors including possible subclinical LV systolic dysfunction, demand ischaemia, and increased ventricular filling pressures. The patient responded well to diuresis and short-term non-invasive positive pressure ventilation for the treatment of respiratory distress. Primary maternal cardiac events were more common in women with CAD due to coronary atherosclerosis (figure 1).

The primary adverse outcomes was not associated with maternal age $(p=0.59)$, past/active smoking history $(p=0.91)$, diabetes $(p=0.30)$, revascularisation prior to pregnancy $(p=0.71)$, atherosclerosis/CABG $(p=0.64)$, multivessel disease $(p=0.38)$, thrombophilia $(p=0.34)$, reduced LVEF $(p=0.39)$, aspirin use $(p=0.78)$ or baseline anticoagulation $(p=0.83)$.

Table 3 Timing of adverse maternal cardiac events

\begin{tabular}{|c|c|c|c|}
\hline $\begin{array}{l}\text { Maternal cardiac } \\
\text { diagnosis }\end{array}$ & $\begin{array}{l}\text { Maternal age } \\
\text { (years) }\end{array}$ & $\begin{array}{l}\text { Cardiac } \\
\text { event }\end{array}$ & $\begin{array}{l}\text { Gestation } \\
\text { (weeks) }\end{array}$ \\
\hline \multicolumn{4}{|l|}{ Primary cardiac events } \\
\hline $\begin{array}{l}\text { Presumed coronary } \\
\text { artery spasm and MI }\end{array}$ & 27 & Sudden death & $\begin{array}{l}8 \text { weeks } \\
\text { postpartum }\end{array}$ \\
\hline Coronary atherosclerosis & 42 & $\mathrm{CHF}$ & 33 weeks \\
\hline Coronary thrombus & 32 & ACS & 34 weeks \\
\hline Coronary atherosclerosis & 33 & $\mathrm{MI}$ & $\begin{array}{l}4 \text { weeks } \\
\text { postpartum }\end{array}$ \\
\hline Coronary atherosclerosis & 32 & MI & $\begin{array}{l}8 \text { weeks } \\
\text { postpartum }\end{array}$ \\
\hline \multicolumn{4}{|l|}{ Other cardiac events } \\
\hline Coronary thrombus & 40 & CVA & 5 weeks \\
\hline Coronary atherosclerosis & 34 & Angina & 12 weeks \\
\hline Coronary atherosclerosis & 41 & Angina & 20 weeks \\
\hline Coronary thrombus & 33 & Angina & 21 weeks \\
\hline Coronary fibroelastosis & 27 & Angina & 24 weeks \\
\hline Coronary atherosclerosis & 28 & Angina & 30 weeks \\
\hline Coronary atherosclerosis & 30 & Angina & 32 weeks \\
\hline Coronary atherosclerosis & 36 & Angina & 33 weeks \\
\hline Coronary atherosclerosis & 41 & Angina & 36 weeks \\
\hline Coronary thrombus & 28 & SVT & 39 weeks \\
\hline $\begin{array}{l}\text { Ehlers-Danlos } \\
\text { Syndrome }\end{array}$ & 32 & $\begin{array}{l}\text { Iliac artery } \\
\text { dissection }\end{array}$ & $\begin{array}{l}1 \text { week } \\
\text { postpartum }\end{array}$ \\
\hline Coronary atherosclerosis & 34 & Angina & $\begin{array}{l}4 \text { weeks } \\
\text { postpartum }\end{array}$ \\
\hline
\end{tabular}

Ischaemic cardiac events (new or progressive angina, ACS/MI, ventricular arrhythmia, cardiac arrest) complicated $26 \%$ of the pregnancies (tables 2 and 3). Treatment of angina, which complicated nine pregnancies, included clinical review alone $(n=3)$, new antianginal therapy $(n=2)$ and hospital admission with serial cardiac biomarkers for exclusion of MI $(n=1)$. One woman had a normal stress echocardiogram at 38 weeks gestation. Two women underwent coronary angiography, one at 38 weeks gestation and the other 4 weeks postpartum. All were treated medically without angina recurrence, premature onset of labour, or fetal loss.

Women with pre-existing coronary atherosclerosis and/or CABG were at highest risk of ischaemic events (48\% vs $10 \%$, $\mathrm{p}=0.003)$. Other complications included supraventricular tachycardia in one woman and left frontal lobe embolic stroke in another; both women had a history of coronary thrombus (table 3). One woman with Ehlers-Danlos syndrome type IV (vascular type) developed bilateral dissection of the external iliac arteries 1 week postpartum.

\section{Obstetric outcomes}

Sixty-two percent of the deliveries were vaginal. Adverse obstetric outcomes occurred in $16 \%(n=8)$ of the pregnancies (table 4 and figure 1): pre-eclampsia in four women and postpartum haemorrhage in four women. Three of the four women diagnosed with pre-eclampsia had proven coronary atherosclerosis and two of four women in whom postpartum haemorrhage occurred were receiving anticoagulants for coronary thrombus. There were no deaths due to obstetric complications.

\section{Fetal and neonatal outcomes}

The median gestational age at live delivery was 38 weeks and median birth weight was $3050 \mathrm{~g}$. Fifteen of 50 (30\%) pregnancies were complicated by an adverse fetal/neonatal event (table 4 and figure 1). Three pregnancies ended in spontaneous abortion ( $<20$ weeks gestation). One pregnancy ended in fetal

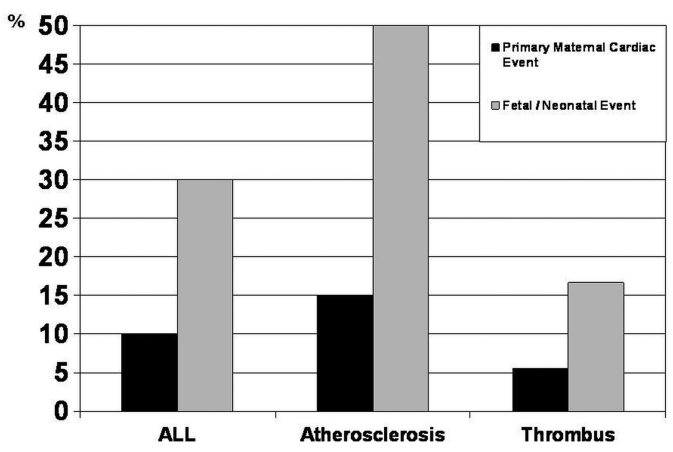

Figure 1 Frequency of maternal cardiac and fetal/neonatal events during pregnancy in women pre-existing coronary artery disease or following an acute coronary syndrome. 
Table 4 Adverse obstetric, fetal and neonatal outcomes

\begin{tabular}{lc}
\hline & $\begin{array}{l}\text { Pregnancies } \\
\mathbf{n}(\%)\end{array}$ \\
\hline Adverse obstetric outcomes & \\
Total events & $8(16)$ \\
Pre-eclampsia & $4(8)$ \\
Postpartum haemorrhage & $4(8)$ \\
Non-cardiac death & $0(0)$ \\
Adverse fetal/neonatal outcomes* & \\
Total events & $15(30)$ \\
Preterm delivery (<37 weeks) & $7(14)$ \\
Low birth weight & $2(4)$ \\
Intrauterine growth retardation & $1(2)$ \\
Intraventricular haemorrhage & $0(0)$ \\
Respiratory distress & $0(0)$ \\
Spontaneous abortion* & $3(6)$ \\
Fetal death & $1(2)$ \\
Neonatal death & $1(2)$ \\
\hline *Spontaneous abortion refers to miscarriage before 20 weeks gestation. \\
tOne pregnancy ended with fetal death due to twin-twin transfusion syndrome. \\
‡Neonatal death occurred 40 days postpartum in an infant born with Trisomy 18.
\end{tabular}

death in utero due to twin-twin transfusion syndrome. There was also one fetal death 40 days postpartum in an infant born with trisomy 18 . Preterm delivery ( $<37$ weeks) occurred in 7 of $50(14 \%)$ pregnancies. Low birth weight $(<2500 \mathrm{~g})$ was documented in two pregnancies and intrauterine growth retardation in one. Adverse fetal/neonatal events occurred in 10 of the 20 women with coronary atherosclerosis $(50 \%)$ and in four of the 18 with a history of coronary thrombosis/embolism (17\%).

\section{DISCUSSION}

In developed nations, heart disease is the leading indirect cause of maternal death in pregnancy. ${ }^{14}$ One specific cardiac condition seen with increasing prevalence in women of childbearing age is coronary disease. Despite an increasing number of young women with this condition, the risk of pregnancy in this group of women has not been well defined. In this study, we found that women with pre-established CAD or an ACS/MI prior to pregnancy were at risk for serious adverse maternal cardiac events (10\% of pregnancies) during pregnancy. The highest rates of non-fatal ischaemic cardiac complications during pregnancy were experienced by women with atherosclerotic coronary disease.

Case reports and case series of pregnancy risk in women with MI prior to pregnancy report maternal mortality ranging between $0 \%$ and $23 \% .^{7-9}$ The largest and most contemporary report describes 20 women included in the European Registry on Pregnancy and Heart Disease with an MI prior to pregnancy, in whom only one experienced new ACS in pregnancy and no mortality was observed. ${ }^{9}$ We observed one maternal death in a woman with a history of MI and moderate LV dysfunction, who died suddenly at home 2 weeks postpartum. In this case, it is difficult to differentiate whether this event occurred secondary to the haemodynamic stress of pregnancy or was simply a manifestation of the natural history of LV dysfunction. Compared with earlier studies, ${ }^{7} 8$ maternal mortality was significantly lower in this study. A number of factors may explain this. All were followed in tertiary cardiac care programmes in the contemporary era, the underlying aetiology of CAD/ACS/MI was identified and treated in most, many had undergone coronary revascularisation and almost all women had preserved ventricular function.

Existing tools for assessing global maternal cardiac risk in pregnancy are the CARPREG and ZAHARA risk scores. ${ }^{12} 13$ These risk scores incorporate a range of clinical predictors including previous cardiac events (arrhythmia, heart failure), poor NYHA functional class, cyanosis, left heart obstruction and the presence of a mechanical valve. These risk scores, derived from cohorts of women with structural and congenital heart disease, were not developed to predict adverse outcomes in women with pre-established CAD or a history of ACS/MI. We found that a pre-existing diagnosis of coronary atherosclerosis was helpful in identifying those at highest risk of cardiac ischaemic events in pregnancy. It is likely that other variables, such as ischaemia prior to pregnancy, multivessel disease or smoking, may better predict outcomes. Disease-specific complications remain important when stratifying risk, as illustrated by the patient in this study with Ehlers-Danlos syndrome whose pregnancy was complicated by vascular dissection. Sudden cardiac death in one woman with ischaemic cardiomyopathy is a reminder of the potential maternal morbidity and mortality associated with significant ventricular dysfunction. ${ }^{9} 14$

Obstetric, fetal and neonatal adverse events were common. Pre-eclampsia and low birth weight appeared to be more common in women with vascular disease (atherosclerosis and/or diabetes). This relationship may be a manifestation of the increased incidence of placental disease in women with systemic vascular disease and endothelial dysfunction. ${ }^{15} 16$ We have previously compared the frequency of adverse neonatal outcomes in pregnant women with and without heart disease, ${ }^{12}$ who were prospectively matched at the time of study enrolment. Neonatal adverse events were much more frequent in women with heart disease versus controls ( $18 \%$ vs $7 \%$, respectively). In the present study, we observed a higher rate of adverse neonatal events (30\%), which was primarily driven by early preterm labour and low birth weight/intrauterine growth restriction. Important differences exist in relation to this and our earlier study cohort, most notably a low prevalence of CAD $(0.02 \%)$ and a predominance of women with congenital and valvular heart disease in the previous study. While the rate of neonatal complications in the current study is almost twice as high as that observed among women with congenital/valvular heart disease, it is premature to conclude that women with $\mathrm{CAD}$ or ACS/MI have worse neonatal outcomes. However, the increased rate of neonatal complications in this contemporary cohort of women with CAD or $\mathrm{ACS} / \mathrm{MI}$ is concerning and deserves further research.

\section{Limitations}

The study has many limitations inherent to a retrospective observational study including small cohort size and the absence of a prospectively matched control group with which outcomes could be compared. Women included in this study were treated in maternity programmes linked to tertiary cardiac care centres, increasing the potential for referral bias. Women with less severe heart disease may not have been referred for specialist care. Women with more severe heart disease may have been advised against pregnancy. To attempt to minimise the impact of referral and/or institutional bias in care and patient outcomes, this study included women from two countries, Canada and the Netherlands. Despite this, outcomes may not reflect those achieved in less specialised centres and in lower-resource settings. $^{9}$

In our study, age was not a major determinant of adverse events. This differs from other studies of MI in pregnancy 
which have reported that the risk of MI rises incrementally with maternal age, ${ }^{17}$ so that by the age of 40 years, women have a 30 -fold higher risk of cardiac ischaemia during pregnancy compared with women aged younger than 20 years. ${ }^{5}$ It is possible that we failed to detect this association because of our relatively small cohort size.

In this study, the interval between previous $\mathrm{MI}$ and the onset of pregnancy was often not available, although this is an important determinant of outcome. ${ }^{6}$ The risk of MI has also been reported to differ by race or ethnicity, black women reported to have the highest risk. ${ }^{5}$ Racial differences in maternal cardiac risk were not a focus of this study, but may be important. An incidental observation was that women enrolled from The Netherlands had a high rate of factor V Leiden deficiency associated with intracoronary thrombus. To identify predictors of outcomes in this patient population, prospective studies in larger sample sizes are needed.

\section{CONCLUSION}

Women with a pre-existing CAD or following ACS/MI are at increased risk of adverse maternal cardiac events in pregnancy. They are also at increased risk of obstetric, fetal and neonatal complications. The presence of coronary atherosclerosis identi-

\section{Key messages}

What is already known on this subject?

- The physiological changes of pregnancy potentially contribute to myocardial ischaemia and other serious cardiac complications during pregnancy. This risk is most relevant for women with coronary disease. While many women with pre-existing coronary artery disease or a history of acute coronary syndromes will consider pregnancy, the pregnancy risk for women with these conditions has not been studied.

- Understanding the pregnancy risk is particularly important because if ischaemic events such as acute myocardial infarction occur during pregnancy, they are associated with high maternal and fetal mortality.

\section{What might this study add?}

- In this multicentre study, we found that there is serious morbidity and mortality for women with pre-existing coronary artery disease/acute coronary syndromes. We further identified risk factors to identify those women at highest risk.

\section{How might this impact on clinical practice?}

- This is the first study to report pregnancy outcomes in this growing population, the study results of which will be helpful for the clinician, aiding in preconception counselling and risk stratification. fies women at highest risk of cardiac ischaemic events during subsequent pregnancy. This information will be helpful for the clinician; aiding in preconception counselling and risk stratification.

Contributors All co-authors contributed to planning, conduct and reporting of the work described in the article. All co-authors contributed to data collection, review and approval of the research contained within this manuscript. CKS (PI) and LJB are responsible for the overall content as guarantor(s).

Funding This research was funded in part by the Beth Medjuck Research Fellowship at the Toronto Congenital Cardiac Centre for Adults, University of Toronto.

\section{Competing interests None.}

Ethics approval Toronto General Hospital Research Ethics Board.

Provenance and peer review Not commissioned; externally peer reviewed.

\section{REFERENCES}

1 Cantwell R, Clutton-Brock T, Cooper G, et al. Saving Mothers' Lives: Reviewing maternal deaths to make motherhood safer: 2006-2008. The Eighth Report of the Confidential Enquiries into Maternal Deaths in the United Kingdom. BJOG 2011;118(Suppl 1):1-203.

2 Gelson E, Gatzoulis MA, Steer $P$, et al. Heart disease - why is maternal mortality increasing? BJOG 2009;116:609-11.

3 Regitz-Zagrosek V, Blomstrom Lundqvist C, Borghi C, et al. ESC Guidelines on the management of cardiovascular diseases during pregnancy: the Task Force on the Management of Cardiovascular Diseases during Pregnancy of the European Society of Cardiology (ESC). Eur Heart J 2011;32:3147-97.

4 Ladner HE, Danielsen B, Gilbert WM. Acute myocardial infarction in pregnancy and the puerperium: a population-based study. Obstet Gynecol 2005;105:480-4.

5 James $\mathrm{AH}$, Jamison MG, Biswas MS, et al. Acute myocardial infarction in pregnancy: a United States population-based study. Circulation 2006:113: 1564-71.

6 Roth A, Elkayam U. Acute myocardial infarction associated with pregnancy. J Am Coll Cardiol 2008;52:171-80.

7 Dufour PH, Occelli B, Puech F. Pregnancy after myocardial infarction. Int J Gynaecol Obstet 1997:59:251-3.

8 Bagg W, Henley PG, Macpherson P, et al. Pregnancy in women with diabetes and ischaemic heart disease. Aust N Z J Obstet Gynaecol 1999:39:99-102.

9 Roos-Hesselink JW, Ruys TP, Stein Jl, et al. Outcome of pregnancy in patients with structural or ischaemic heart disease: results of a registry of the European Society of Cardiology. Eur Heart J 2013;34:657-65.

10 Thygesen K, Alpert JS, Jaffe AS, et al. Third universal definition of myocardial infarction. J Am Coll Cardiol 2012;60:1581-98.

11 Lang RM, Bierig M, Devereux RB, et al. Recommendations for chamber quantification: a report from the American Society of Echocardiography's Guidelines and Standards Committee and the Chamber Quantification Writing Group, developed in conjunction with the European Association of Echocardiography, a branch of the European Society of Cardiology. J Am Soc Echocardiogr 2005;18:1440-63.

12 Siu SC, Sermer M, Colman JM, et al. Prospective multicenter study of pregnancy outcomes in women with heart disease. Circulation 2001;104:515-21.

13 Drenthen W, Boersma E, Balci A, et al. Predictors of pregnancy complications in women with congenital heart disease. Eur Heart J 2010;31:2124-32.

14 Grewal J, Siu SC, Ross HJ, et al. Pregnancy outcomes in women with dilated cardiomyopathy. J Am Coll Cardiol 2009;55:45-52.

15 Chambers JC, Fusi L, Malik IS, et al. Association of maternal endothelial dysfunction with preeclampsia. JAMA 2001;285:1607-12.

16 Sattar N, Ramsay J, Crawford L, et al. Classic and novel risk factor parameters in women with a history of preeclampsia. Hypertension 2003;42:39-42.

17 Bush N, Nelson-Piercy C, Spark P, et al. Myocardial infarction in pregnancy and postpartum in the UK. Eur J Prev Cardiol 2013;20:12-20. 